(5) $\therefore f^{\prime}(x)$ will be $>0$ just before and $<0$ just after $x=a$,

(6) $\therefore f(x)$ will be increasing......., decreasing.........

(7) i.e. $f(a)$ will be a maximum.

Again, if $f^{\prime}(a)=0$ and $f^{\prime \prime}(a)=0$ and a maximum of $f^{\prime \prime}(x)$, then $f^{\prime \prime}(x)$ is necessarily $<f(a)$, i.e. $<0$, in the neighbourhood of $x=\dot{a}$. Hence (3), $(4),(5),(6)$ and (7) apply in this case also.

We conclude that if $f^{\prime \prime}(a)$ is negative or zero and a maximum of $f^{\prime \prime}(x)$, and if $f^{\prime}(a)=0$, then $f(a)$ is a maximum value. ..... (8)

Hence if we suppose $f^{(r)}(a)=0$, for the values $1,2,3, \ldots 2 n-1$ of $r$ and $f^{(2 n)}(a)<0,(8)$ enables us to say that $f^{(2 n-2)}(a)$ is a maximum, and thus that $f^{(2 n-4)}(a)$ is a maximum, and so on, till it is proved that $f(a)$ is a maximum value.

Thus a necessary and sufficient condition for $f(a)$ being a maximum value in the case of a function $f(x)$ which, with those of its derivatives which come into the statement, is continuous in the neighbourhood of $x=a$, is :-

(9) The first of the functions $f^{\prime}(a), f^{\prime \prime}(a), f^{\prime \prime \prime}(a), \ldots$ which does not vanish must be of even order and negative.

To extend the theory to minima.

If $f(a)$ is a minimum of $f(x)$, then $-f(a)$ is a maximum of $-f(x)$. Hence for a minimum value of $f(a)$ we have only to change the word negative into positive in condition (9).

The chief interest of the foregoing lies in the fact that the general form of criterion for a maximum or minimum of $f(x)$ has hitherto been deduced by the aid of Taylor's Theorem. Since questions on maxima and minima are amongst the most interesting and practically valuable applications of the Differential Calculus, it may be useful for teachers to have a proof of the criterion in question not requiring Taylor's Theorem.

R. F. Mutrhead.

\title{
A Footnote to Euclid, Book III.
}

The following two propositions are converses of Euclid HII., 11 and 12.

Prop. 1. If the circumferences of two circles whose centres are $A$ and $B$ have a common point $C$ lying in the straight line $A B$, then the circles touch each other externally at $C$. 
For if $P$ be a point other than $C$ on the circumference of the circle whose centre is $B$, and if $A P$ and $P B$ be joined,

$$
\begin{aligned}
A P+P B & >A B, \\
& >A C+C B, \\
& >A C+P B, \text { since } C B=P B . \\
\therefore A P & >A C .
\end{aligned}
$$

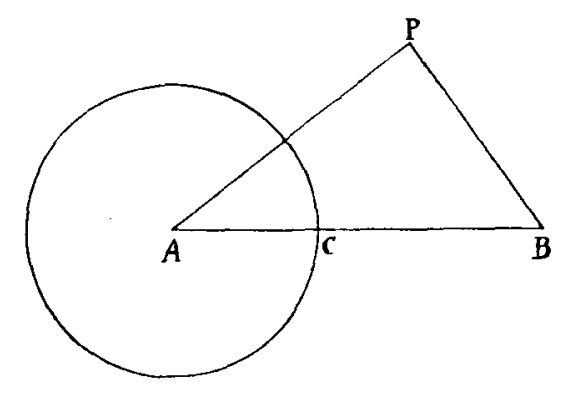

$\therefore P$ lies without the circle whose centre is $A$, i.e. every point of the oircumference of the circle whose centre is $B$, excepting $C$, lies outside of the circle whose centre is $A$.

Hence the circles do not cut one another.

Hence, according to Euclid's definition of tangency of circles, the two circles touch each other.

Prop. 2. If the circumferences of two circles whose centres are $A$ and $B$ have a common point $C$ in $A B$ produced, the circles touch each other, that whose centre is $B$ being internal to the other.

The proof need not be given, as it is on exactly the same lines as the proof of the preceding proposition.

It may be noted that in Euclid's definitions of tangency, both of a line and a circle, and of two circles, the phrase "do not cut one another" occurs. This is a negative condition, which renders immediate application of these definitions to deductions involving tangency somewhat inconvenient. So far as tangency by a straight line is concerned, Euclid's Proposition III., 16 deduces a positive characteristic peculiar to a tangent at a point $C$ on the circumference of a circle, viz., the fact that it is at right angles to the diameter of which $C$ is one extremity; and this is made the basis of all the subsequent proofs involving tangency of a straight line. 
The propositions 1 and 2 given above may be taken to furnish a similiar help in the theory of tangent circles, i.e. by affording a positive criterion of the existence of tangency.

Euclid has but few propositions dealing with tangent circles, and for these the propositions of which the two given are converses suffice. But there are many geometrical problems or exercises in which Propositions 1 and 2 can be usefully applied. We may take as an example one of the solutions of that special case of the Apollonian contact problem where it is required to construct a circle to pass through a given point, and to touch a given circle and a given straight line.

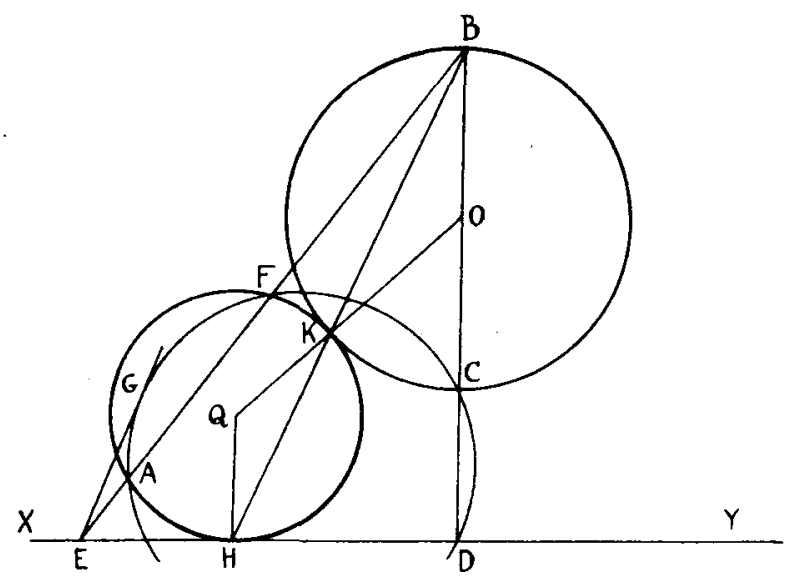

Let $A$ be the given point, and $B C$ that diameter of the given circle which is perpendicular to the given straight line $X Y$.

Let $B C$ produced meet $X Y$ in $D, B A$ produced meet $X Y$ in $E$.

Let the circle $D C A$ meet $B E$ in $F$.

Let $E G$ be tangent to the circle $D C A$.

From $E Y$ (or $E X$ ) cut off $E H$ equal to $E G$.

Let $B H$ cut the given circle in $K$.

Then since $B \widehat{K} C=90^{\circ}=C \widehat{D} H, C D H K$ is a eyclic quadrilateral.

$\therefore B K . B H=B C . B D=B F . B A$.

$\therefore K H F A$ is a cyclic quadrilateral. Let $Q$ be the centre of its circumscribing circle, which is the required circle.

For $E H^{2}=E G^{2}=E A . E F$. Hence this circle touches the given line. 
Let $O$ be the centre of the given circle and $Q$ that of the circle HKFA.

Join $Q K, O K . \quad O K B=O B K=K \widehat{H} Q=H K Q$.

$\therefore Q K$ and $K O$ are in the same straight line.

Hence by Prop. 1 the circles whose centres are $O$ and $Q$ touch externally at $C$.

Another solution is got by taking $E H$ in the opposite direction. Two more solutions, with internal contact, are got by interchanging $B$ and $C$ in the figure.

If $A$ lies within the given circle, or if the given circle and $A$ are on opposite sides of $X Y$, there is no solution.

If $X Y$ cuts the given circle there are in general two solutions, both internal, or both external.

R. F. Muirhead.

\section{On the Treatment in School Texts of the Sphere, Cone and Pyramid.}

An important note in the Proc. Edin. Math. Soc. of May 1904 pointed out that the then prevalent School method of tangents made it difficult for the pupil to gain sound knowledge in his later studies. The same criticism may, I submit, be directed against the treatment, now prevalent in school texts, of (1) surface of sphere, (2) volume of sphere, and (3) volume of cone and pyramid.

"Area of sphere..... Two planes cut the diameter $A O B$ perpendicularly at $M$ and $N$, and intercept the arc $P Q$. Revolving about the axis $A O B$, the arc $P Q$ generates a belt of a sphere, while $p q$ generates a corresponding belt of the circumscribing cylinder. The planes are supposed to be near together. $A s P Q$ is short we may suppose it to be a straight line; and the surface generated by $P Q$ is the curved surface of a frustum of a cone, and hence, from a previous formula, equals $2 \pi \times P Q \times$ half-way perpendicular; hence etc. in the usual way, surface of the $P Q$-belt of the sphere equals surface of corresponding $p q$-belt of cylinder."

From this position advance is made to total surface of sphere.

The above, quoted almost verbatim from a 1926 textbook by a first-rate publisher, is a fair sample of the now prevailing treatment 